09

\title{
Схема VLS-монохроматора высокого разрешения для синхротронного излучения
}

\author{
(C) А.Н. Шатохин, ${ }^{1}$ Е.А. Вишняков, ${ }^{1}$ А.О. Колесников, ${ }^{1}$ А.Д. Николенко, ${ }^{2,3}$ Е.Н. Рагозин ${ }^{1}$ \\ ${ }^{1}$ Физический институт им. П.Н. Лебедева РАН, \\ 119991 Москва, Россия \\ ${ }^{2}$ Институт ядерной фризики им. Г.И. Будкера СО РАН, \\ 630090 Новосибирск, Россия \\ ${ }^{3}$ Институт катализа им. Г.К. Борескова СО РАН, \\ 630090 Новосибирск, Россия \\ e-mail: shatohinal@gmail.com
}

Поступило в Редакцию 29 марта 2021 г.

В окончательной редакции 29 марта 2021 г.

Принято к публикации 29 марта 2021 г.

Рассчитан монохроматор высокого разрешения для широкой спектральной области 125-4200 Азмерительного канала для проектируемого источника синхротронного излучения четвертого поколения „СКИФ“ (г. Новосибирск). Оптическая схема монохроматора включает вогнутое зеркало скользящего падения, плоскую VLS-решетку скользящего падения и выходную щель. Предполагается использовать две сменные VLS-решетки для поддиапазонов 125-1000 А и 900-4200 А с частотами штрихов в центре решеток 600 и $150 \mathrm{~mm}^{-1}$ соответственно. Перестройка длины волны в каждом из двух диапазонов происходит только за счет поворота VLS-решетки. За счет выбора коэффициентов $p_{1}$ VLS-решеток, фокусное расстояние изменялось во всем спектральном диапазоне незначительно, а за счет выбора коэффициентов $p_{2}$ VLS-решеток компенсируются аберрации и зеркала, и решетки. Разрешающая способность схемы, полученная с помощью численной трассировки лучей, превышает 1000 в диапазоне $125-1000 \AA$ и 2000 в диапазоне $900-4200 \AA$.

Ключевые слова: вакуумный ультрафиолет, спектроскопия, VLS-решетка, синхротронное излучение.

DOI: $10.21883 / J T F .2021 .10 .51369 .86-21$

\section{Введение}

Сибирский кольцевой источник фотонов „СКИФ“ представляет собой источник синхротронного излучения 4-го поколения и предназначен для проведения широкого спектра работ с использованием рентгеновского и вакуумного ультрафиолетового (ВУФ) излучения в интересах различных отраслей науки и техники. Как ожидается, комплекс будет введен в эксплуатацию в 2024 году. Первая очередь источника включает в себя шесть станций синхротронного излучения, большая часть которых предназначена для работы с жестким рентгеновским изучением. Только одна станция из шести станций первой очереди (станция 1-6, „Электронная структура“, [1]) будет ориентирована на работу с мягким рентгеновским и ВУФ-излучением в спектральном диапазоне $10-2000 \mathrm{eV}$. Производительности этой станции будет явно недостаточно для покрытия экспериментальных потребностей в области ВУФ и мягкого рентгеновского диапазона, и поэтому во второй очереди должны быть предусмотрены соответствующие станции. В связи с закономерным дефицитом экспериментальных мощностей на первом этапе станция 1-6 проектировалась как установка с максимально широким спектральным охватом. В станциях второй очереди представляется целесообразным уменьшить спектральный охват каждой из новых станций за счет улучшения других потре- бительских характеристик. Так, среди станций второй очереди предусмотрена, по меньшей мере, одна станция ВУФ-диапазона для работы в спектральном диапазоне 3-100 eV, которая по сравнению со станцией 1-6 будет иметь больший акцептанс, и, как следствие, позволит получать больший поток фотонов в своем рабочем спектральном диапазоне. Предполагается, что эта станция будет получать излучение из поворотного магнита накопителя и на ней будет реализована методика изучения кинетики горения различных объектов с использованием масс-спектрометрии с фотонной ионизацией продуктов горения. Кроме того, как ожидается, на этой станции будет реализован ряд метрологических методик для различных потребителей. Предлагаемый нами монохроматор рассчитан для использования на этой станции.

Передовые исследования, проводимые на синхротронах третьего и четвертого поколений, часто требуют выделения монохроматического излучения высокой спектральной чистоты, а также быстроты и удобства перестройки канала излучения на другую длину волны. При использовании дифракционного монохроматора высокого разрешения для выделения монохроматического излучения ВУФ-диапазона спектра из полихроматического пучка синхротронного излучения (СИ), крайне желательно сохранять постоянным фокусное расстояние монохроматора и направление выхода дифрагирующего излучения при перестройке рабочей длины волны. 
В этом случае положение выходной щели остается неизменным и минимизируется число перемещений элементов в вакууме. Такая задача может быть решена, во-первых, в модифицированной схеме Черни-Тернера c применением плоской отражающей дифракционной решетки, работающей в параллельном пучке, и двух вогнутых зеркал скользящего падения (желательно внеосевых параболических/эллиптических цилиндров), служащих для коллимации и последующей фокусировки излучения [2]. Во-вторых, эта задача решается в схеме Хеттрика-Андервуда с использованием фокусирующего зеркала и плоской VLS-решетки, ${ }^{1}$ работающей в сходящемся пучке $[3,4]$. Обе схемы объединяет простота: длина волны перестраивается за счет поворота только дифракционной решетки. Однако в схеме VLS-монохроматора Хеттрика-Андервуда используется всего два оптических элемента, а достижение высокой и сверхвысокой $\left(\lambda / \delta \lambda>10^{4}\right)$ разрешающей способности не сопряжено с использованием асферической оптики скользящего падения.

Следует отметить, что есть и другие оптические схемы монохроматоров высокого разрешения с неподвижной щелью, сохраняющие направление выхода дифрагирующего излучения (см., например, [5]). В [5] используется цилиндрическое зеркало, плоская решетка и вспомогательное плоское зеркало, причем сканирование осуществляется поворотом решетки, согласованным с поворотом и одновременной трансляцией плоского зеркала. Недостатком таких схем является большое число оптических элементов, а также определенные сложности с перестройкой рабочей длины волны. Примеры схем приборов со сложной кинематикой оптических элементов можно найти в обзорах, например, [6,7].

В настоящее время VLS-спектрометры и VLS-монохроматоры различного типа широко применяются в исследованиях в мягком рентгеновском (МР) диапазоне спектра $[6,7]$. Они используются в спектроскопии и диагностике лабораторной плазмы [8], астрофизике [9-11], рефлектометрии/метрологии с использованием лазерноплазменного источника МР излучения [12], рентгеновском флуоресцентном анализе с использованием СИ и эмиссионной спектроскопии, совмещенной с электронным микроскопом [13]. Пользовательские каналы МР/ВУФ-излучения на зарубежных синхротронах часто используют монохроматоры и спектрометры на основе VLS-решеток [14-16]. Активно идет разработка специализированных VLS-спектрометров для исследования электронной структуры различных материалов и молекул методом спектроскопии резонансного неупругого рентгеновского рассеяния (RIXS) под действием СИ [17-19].

Свойство решеток с монотонно меняющимся шагом (изменять спектральную фокальную кривую) было отмечено еще М. Корню в 1893 году [20]. Однако после

\footnotetext{
${ }^{1}$ Отражательные дифракционные решетки с шагом, монотонно меняющимся на апертуре по заданному закону (Varied Line-Space Gratings), принято называть VLS-решетками.
}

этого еще почти в течение века усилия изготовителей решеток были направлены на поддержание максимальной эквидистантности штрихов, так как это ассоциировалось с высоким качеством решеток. В 1970-1980е годы к идее использовать фокусирующие свойства апериодических решеток обратились Т. Харада [9,21-24], М. Хеттрик и Дж. Андервуд [3,4,25]. Сканирующие спектрометры/монохроматоры с плоской VLS-решеткой (спектрометры Хеттрика) получили широкое распространение и стали коммерчески доступными [25]. Для изготовления VLS-решеток используют программируемые гравировальные станки, интерференционную литографию, а также электронно-лучевую литографию (см. обзоры $[6,7])$.

В последние годы в России была разработана технология изготовления плоских и вогнутых VLS-peшеток методом интерференционной литографии, включающая расчет оптической схемы интерференционной литографии, и был накоплен опыт создания VLS-спектрометров $[6,7,26,27]$. Учитывая это обстоятельство, а также широкие возможности подхода Хеттрика-Андервуда, мы остановились именно на этом подходе при расчете схемы VLS-монохроматора для ВУФ канала на накопителе 4-го поколения „СКИФ“.

\section{1. Постановка задачи}

Предлагается VLS-монохроматор для СИ в диапазоне 125-4200 А на основе плоской VLS-решетки скользящего падения, работающей в сходящемся пучке. Особенности канала СИ задают геометрию схемы: расстояние от поворотного магнита синхротрона до оптических элементов монохроматора постоянно и составляет $\sim 28 \mathrm{~m}$; оптические элементы должны располагаться компактно; расстояние от оптических элементов до выходной щели также постоянно и равно $\sim 1200 \mathrm{~mm}$; направление выхода излучения из щели должно быть постоянным. Желаемая разрешающая способность должна составлять $\lambda / \delta \lambda>10^{3}$ во всем спектральном диапазоне.

В качестве монохроматора принята следующая схема (рис. 1). Излучение из практически точечного источника фокусируется вогнутым сферическим зеркалом скользящего падения, при этом горизонтальный фокус пучка находится на расстоянии, примерно равном расстоянию

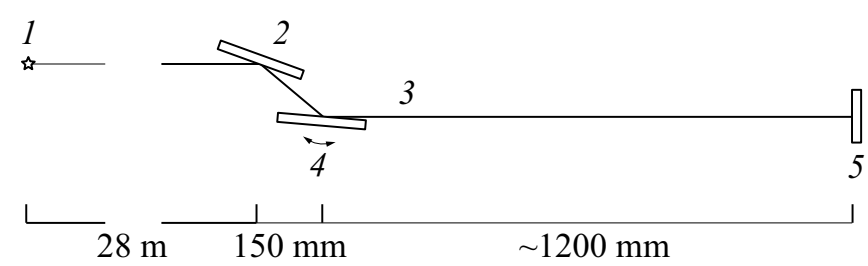

Pис. 1. Схема монохроматора на основе плоской VLSрешетки: 1 - источник, 2 - вогнутое зеркало, 3 - сменная VLS-решетка, 4 - перестройка длины волны осуществляется простым поворотом решетки, 5 - выходная щель. 
до выходной щели. Установка зеркала далека от роуландовской и является сильно асимметричной. После зеркала сходящийся пучок падает на плоскую VLSрешетку и дифрагирует в первом внешнем порядке. Для удобства использования схема не отклоняет падающее излучение: угол отклонения решеткой равен по величине и противоположен по знаку углу отклонения зеркалом. За счет использования VLS-решетки излучение фокусируется в области выходной щели, а изменение длины волны происходит только за счет простого поворота решетки. Схема спектрометра похожа на схему Хеттрика-Андервуда $[3,4]$, однако отличается от нее сильно асимметричной установкой фокусирующего зеркала.

\section{2. Расчет схемы}

Рассмотрим плоскую дифракционную решетку с частотой штрихов, меняющейся по закону $p(w)$, где $w-$ координата на апертуре решетки, перпендикулярная штриху (рис. 2). Разложим этот закон в ряд Тейлора по координате $w: p(w)=p_{0}+p_{1} w+p_{2} w^{2}+\ldots$. Тогда $p_{0}$ - частота штрихов в центре апертуры и этот коэффициент отвечает за направление дифрагированного излучения, коэффициент $p_{1}$ отвечает за фокусирующие свойства решетки, а коэффициент $p_{2}$ может компенсировать аберрацию комы дифрагированного пучка [26].

Пусть на эту решетку падает плоский монохроматический пучок с длинной волны $\lambda_{1}$ под скользящим углом $\varphi_{1}$ и дифрагирует в порядке $m$ под скользящим углом $\psi_{1}$. Тогда величины связаны уравнением дифракционной решетки (1):

$$
\cos \left(\psi_{1}\right)-\cos \left(\varphi_{1}\right)=m p_{0} \lambda_{1}
$$

При том же положении падающего и дифрагированного пучков повернем решетку на небольшой угол. Пусть теперь на решетку падает плоский монохроматический пучок с длиной волны $\lambda_{2}$ под скользящим углом $\varphi_{2}$ и дифрагирует в порядке $m$ под скользящим углом $\psi_{2}$, причем $\varphi_{1}+\psi_{1}=\varphi_{2}+\psi_{2}=\Omega$. Таким образом, происходит перестройка длины волны при постоянном направлении падающего и дифрагированного излучения. Связь между длиной волны и углом падения излучения на решетку

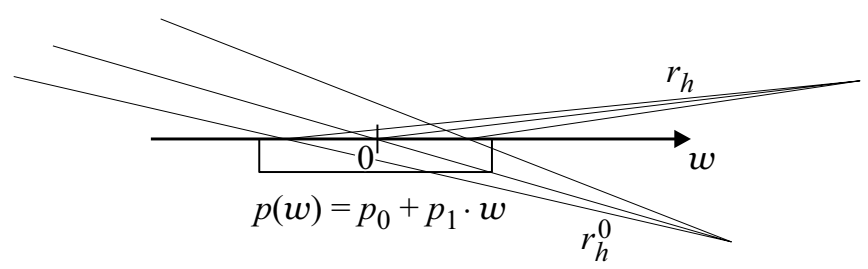

Рис. 2. Изменение фокусного расстояния при дифракции на плоской отражательной VLS-решетке. определяется формулами

$$
\begin{gathered}
\varphi=\frac{\Omega}{2}-\arcsin \frac{m p_{0} \lambda}{2 \sin (\Omega / 2)}, \\
m \lambda=\frac{2 \sin (\Omega / 2) \sin (\Omega / 2-\varphi)}{p_{0}} .
\end{gathered}
$$

Такая схема решает вопрос постоянного направления падающего и дифрагированного излучения. Для решения вопроса о постоянстве фокусного расстояния для дифрагированного излучения будем использовать свойства VLS-решеток.

Пусть на решетку падает сходящийся пучок с длиной волны $\lambda$ и расстоянием от центра решетки до фокуса $r_{h}^{0}$ (рис. 2). Тогда дифрагированное излучение сфокусируется на расстоянии $r_{h}$, определяемом формулой (3):

$$
m \lambda p_{1}=\frac{\sin ^{2} \varphi}{r_{h}^{0}}-\frac{\sin ^{2} \psi}{r_{h}} .
$$

Потребовав выполнения этого условия для двух длин волн $\lambda_{1,2}$ при одинаковых $p_{1}$ и $r_{h}$, в схеме с поворотом решетки, описанной ранее, получим жесткое ограничение на $r_{h}(4)$ :

$$
r_{h}=r_{h}^{0} \frac{\sin ^{2} \psi_{1} \lambda_{2}-\sin ^{2} \psi_{2} \lambda_{1}}{\sin ^{2} \varphi_{1} \lambda_{2}-\sin ^{2} \varphi_{2} \lambda_{1}} .
$$

Также и $p_{1}$ перестает быть свободным параметром (5):

$$
p_{1}=\left(\frac{\sin ^{2} \varphi_{1}}{r_{h}^{0}}-\frac{\sin ^{2} \psi_{1}}{r_{h}}\right) / \lambda .
$$

T. е. при использовании VLS-решетки, у которой коэффициент $p_{1}$ удовлетворяет (5), а угол падения на решетку отвечает (2), получим, что фокусное расстояние одинаково $\left(r_{h}\right)$ для углов падения $\varphi_{1,2}$ и соответствующих им длин волн $\lambda_{1,2}$, и при этом излучение идет в одном направлении для всех длин волн.

\section{3. Выбор параметров схемы}

Из-за большой ширины требуемого спектрального диапазона (125-4200 ̊) при использовании одной дифракционной решетки в схеме монохроматора расфокусировка будет значительной при любом выборе пары длин волн $\lambda_{1,2}$. Поэтому в нашей схеме предполагается использование двух сменных дифракционных решеток.

В качестве двух диапазонов предлагаются примерно равные в логарифмическом масштабе диапазоны 125-1000 А и 900-4200 А. В качестве угла отклонения решеткой (и зеркалом) выбран угол $32^{\circ}$, что соответствует углу падения излучения на зеркало $16^{\circ}$. При таком угле скользящего падения излучения золотое покрытие зеркала отражает более $45 \%$ энергии излучения во всем требуемом спектральном диапазоне. Этот угол является параметром оптимизации схемы. Уменьшение 
этого угла приводит к увеличению коэффициента отражения зеркала и решетки, но при этом падают разрешающая способность и приемный угол прибора. В качестве средней частоты штрихов VLS-решеток выбраны 600 и $150 \mathrm{~mm}^{-1}$.

Для выбора схемы монохроматора необходимо оптимизировать ее параметры по некоторым критериям. В качестве одного из этих критериев выбрана разрешающая способность прибора во всем требуемом диапазоне спектра. Разрешающая способность определяется через ширину изображения источника в плоскости выходной щели $S$ и обратную линейную дисперсию прибора $d \lambda / d s$ через соотношение $\lambda / \delta \lambda=\lambda /(S d \lambda / d s)$. Ширина изображения при этом складывается из геометрической расфокусировки $S_{g}$, аберраций высших порядков $S_{a}$ и дифракционной расходимости $S_{d}$. В качестве критериев удачной оптимизации схемы примем разрешающую способность, обусловленную геометрической расфокусировкой, не менее 5000 во всем выбранном спектральном диапазоне при рабочей ширине VLS-решетки $50 \mathrm{~mm}$, и разрешающую способность, обусловленную аберрациями высших порядков, свыше 1000 во всем диапазоне.

Начнем с более коротковолнового диапазона 125-1000 А, для которого уже определены частота штрихов в центре VLS-решетки $p_{0}=600 \mathrm{~mm}^{-1}$, угол отклонения излучения решеткой $\Omega=32^{\circ}$, расстояние от центра решетки до выходной щели $L_{2}=1200 \mathrm{~mm}$, диапазон 125-1000 , ширина VLS-решетки $50 \mathrm{~mm}$, расстояние от центра решетки до центра фокусирующего зеркала $150 \mathrm{~mm}$. Для максимизации минимальной разрешающей способности во всем диапазоне в качестве длин волн точной фокусировки $\lambda_{1,2}$ выбираем $\lambda_{1}=200 \AA$ и $\lambda_{2}=770 \AA$. При этом потребуется расстояние от центра решетки до фокуса падающего на решетку сходящегося пучка $L_{1} \approx 1201.65 \mathrm{~mm}, p_{1}=0.956 \mathrm{~mm}^{-2}$. Для обеспечения такого падающего на решетку пучка вспомогательное фокусирующее зеркало должно иметь следующие параметры: расстояние от источника до зеркала - $28000 \mathrm{~mm}$, расстояние от зеркала до решетки $150 \mathrm{~mm}$, скользящий угол падения на зеркало $16^{\circ}$, радиус кривизны зеркала $9356 \mathrm{~mm}$.

При этом расстояние от фокуса дифрагированного излучения до выходной щели будет изменяться от нуля до менее чем $4.5 \mathrm{~mm}$ во всем диапазоне, а ширина изображения, обусловленная геометрической расфокусировкой, составит менее $20 \mu \mathrm{m}$ в диапазоне $100-850 \AA$. Обратная линейная дисперсия изменяется от $3.6 \AA / \mathrm{mm}$ на $120 \AA$ до $2.35 \AA / \mathrm{mm}$ на $1000 \AA$, а разрешающая способность составит более 5000 во всем спектральном диапазоне (рис. 3).

Для оценки ширины изображения, обусловленной аберрациями высших порядков, была проведена численная трассировка лучей схемы с помощью программы XOP 2.3 с расширением SHADOW VUI 1.12 [28]. При коэффициенте VLS-решетки $p_{2}=0$, определяющей
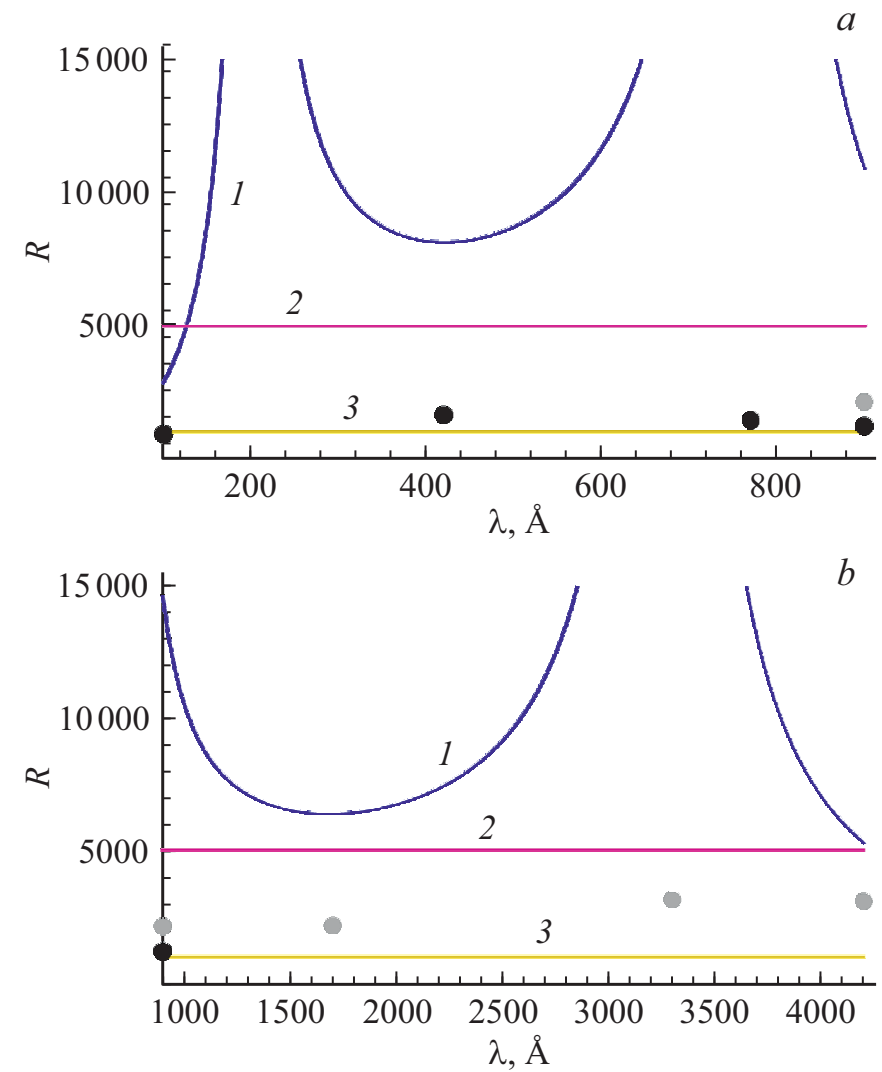

Рис. 3. 1 - расчетная зависимость разрешающей способности монохроматора от длины волны с учетом только геометрической расфокусировки (без учета аберраций) для двух VLS-решеток (диапазоны 125-1000 $(a)$ и 900-4200 $(b)$ ); 2 - разрешающая способность, соответствующая 5000, 3 разрешающая способность, соответствующая 1000. Точками показана разрешающая способность, рассчитанная с помощью численной трассировки лучей (с учетом аберраций).

аберрацией будет кома, из-за которой ширина изображения будет составлять доли миллиметра. Для ее компенсации необходимо выбрать параметр $p_{2}$. Критерием оптимизации служит требование, чтобы разрешающая способность $R$ во всем диапазоне была не менее 1000 . При этом если рассмотреть $R=\lambda / \Delta \lambda$, беря в качестве $\Delta \lambda$ полную (по основанию) ширину изображения, то, выбрав $p_{2}=-1.50 \cdot 10^{-3} \mathrm{~mm}^{-3}$, получим $R=1000-1400$. Причем ширина почти во всем диапазоне все равно определяется комой, а значит, уменьшив ширину решетки в полтора раза, можно увеличить $R$ вдвое.

При переходе к длинноволновому диапазону $900-4200 \AA$ с решеткой с $p_{0}=150 \mathrm{~mm}^{-1}$ неизменными остаются угол отклонения излучения решеткой $\Omega=32^{\circ}$, расстояние от центра решетки до выходной щели $L_{2}=1200 \mathrm{~mm}$, расстояние от центра решетки до фокуса падающего на решетку сходящегося пучка $L_{1} \approx 1201.65 \mathrm{~m}$, ширина решетки $50 \mathrm{~mm}$, а также параметры фокусирующего зеркала скользящего падения. Свободным параметром остается коэффициент $p_{1}$, кото- 

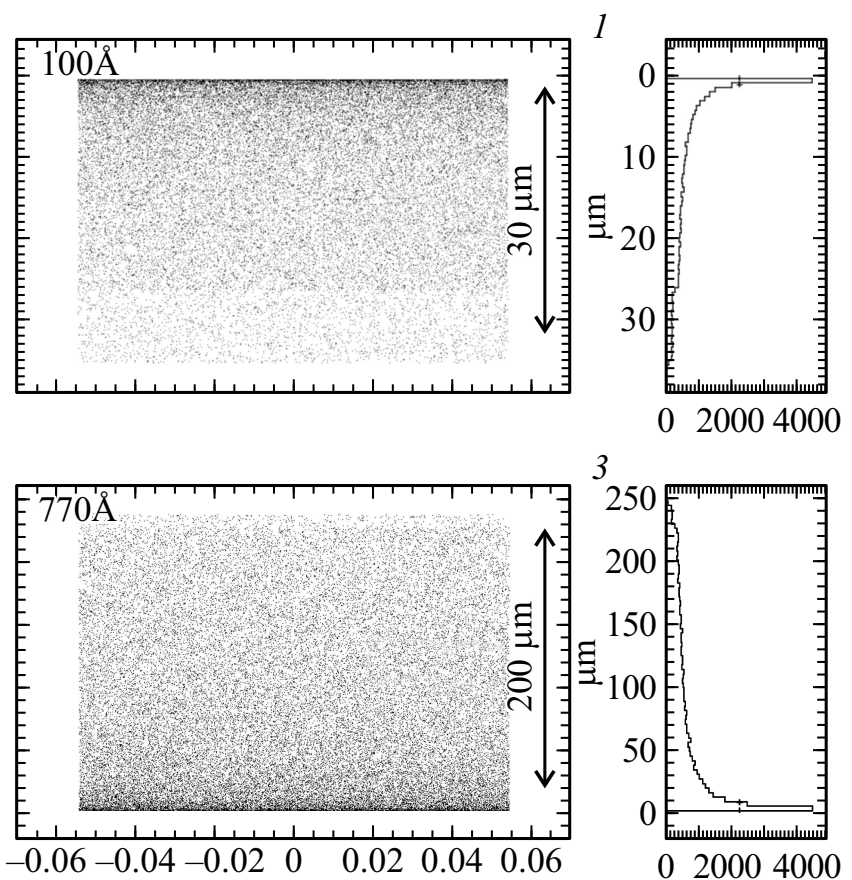

3

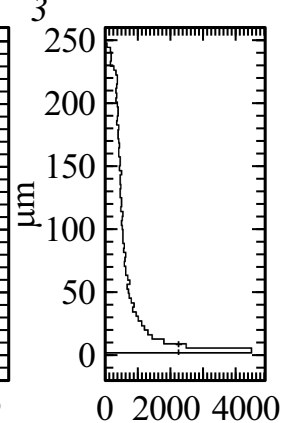

5
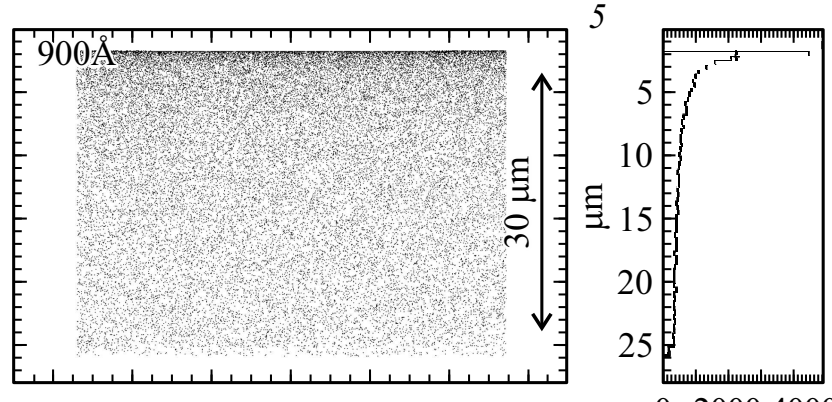

020004000
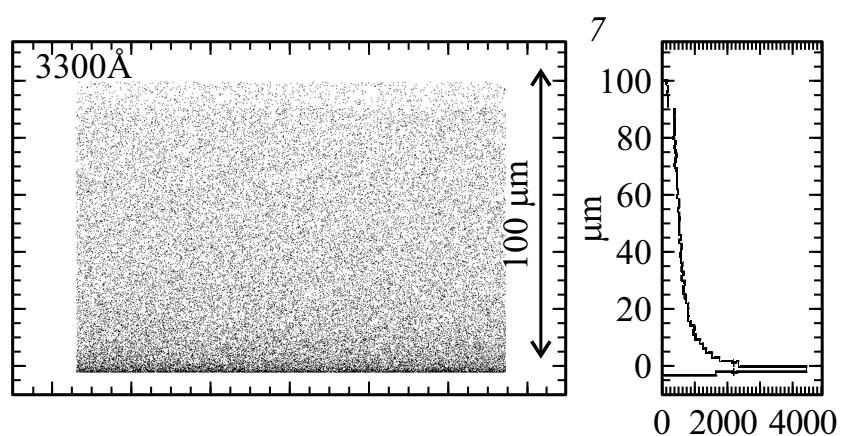
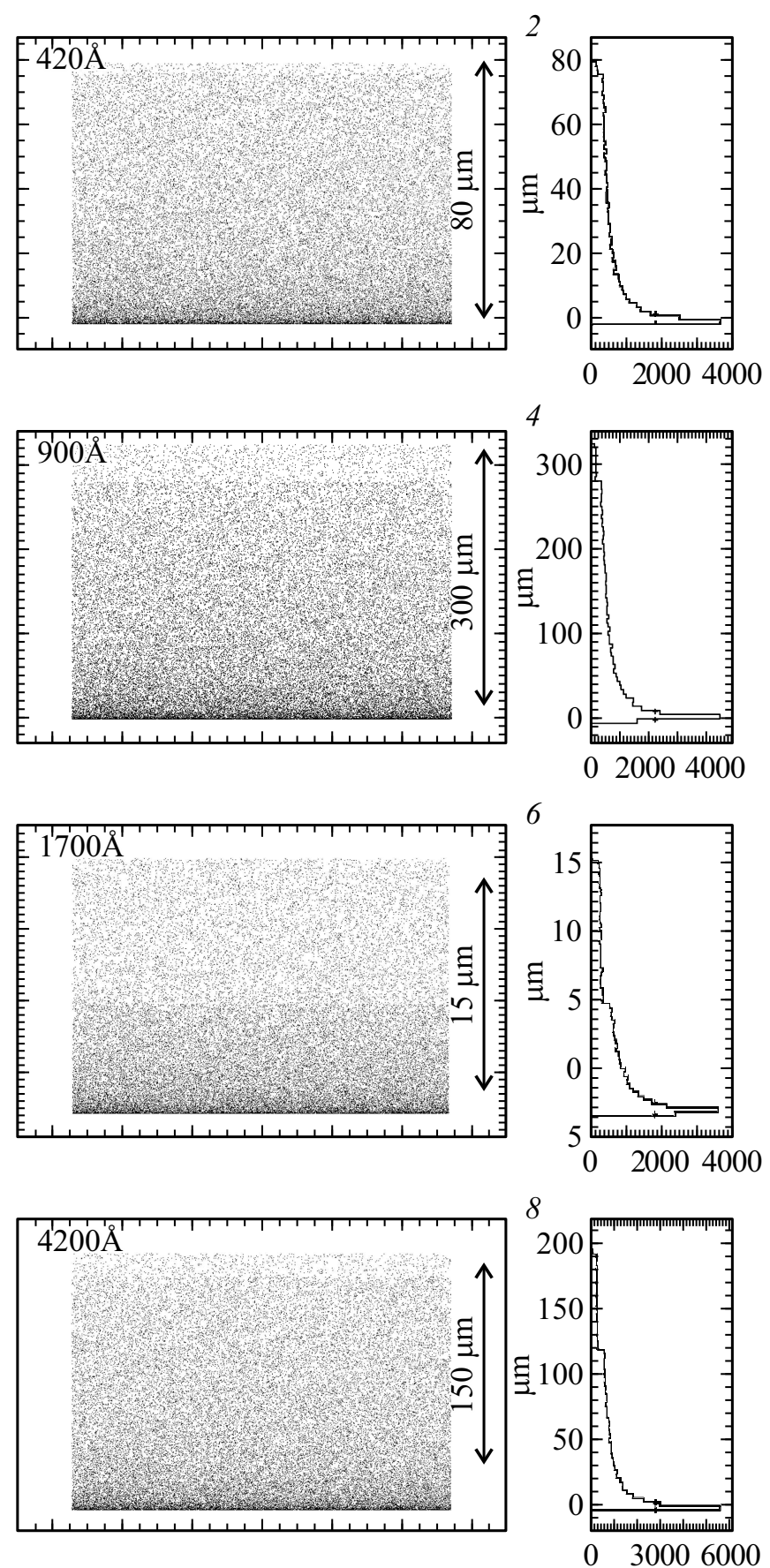

Рис. 4. Изображения спектральных линий точечного монохроматического источника, полученные методом численной трассировки лучей для двух VLS-решеток (диапазоны 125-1000 А (вверху) и 900-4200 А (внизу)).

рый определит длины волн точной фокусировки $\lambda_{1}$ и $\lambda_{2}$. В данном случае, так как параметр $L_{1}$ уже определен, при максимизации минимальной разрешающей способности во всем диапазоне, одна из точек оказывается вне диапазона: $p_{1}=0.24 \mathrm{pcs} / \mathrm{mm}^{2}, \lambda_{1}=726 \AA$ и $\lambda_{2}=3290 \AA$.

Параметры, обусловленные геометрической расфокусировкой, будут следующими: ширина изображения составит менее $40 \mu \mathrm{m}$ в диапазоне 900-3800 ̊, расфокусировка (расстояние от фокуса дифрагированного излучения до выходной щели) составит менее $5 \mathrm{~mm}$ для всех длин волн из рассматриваемого диапазона спектра, обратная линейная дисперсия от $14.0 \AA / \mathrm{mm}$ на $900 \AA$ до $9.1 \AA / \mathrm{mm}$ на $4200 \AA$. Расчетная разрешающая способность составляет более 5000 во всем спектральном диапазоне (рис. 3).

Для оценки ширины изображения, обусловленной аберрациями высших порядков, также была проведена численная трассировка лучей. Выбрав 
Параметры монохроматора

\begin{tabular}{|c|c|c|c|}
\hline \multicolumn{4}{|c|}{ Вогнутое зеркало } \\
\hline \multicolumn{2}{|c|}{ Радиус кривизны, mm } & \multicolumn{2}{|c|}{9356} \\
\hline \multicolumn{2}{|c|}{ Апертура $D, \mathrm{~mm}$} & \multicolumn{2}{|c|}{50} \\
\hline \multicolumn{2}{|c|}{ Угол падения от нормали, } & \multicolumn{2}{|c|}{74} \\
\hline \multicolumn{2}{|c|}{ Расстояние источник-зеркало, mm } & \multicolumn{2}{|c|}{28000} \\
\hline \multicolumn{2}{|c|}{ Расстояние зеркало-фокус, mm } & \multicolumn{2}{|c|}{1352} \\
\hline \multicolumn{2}{|c|}{ Расстояние зеркало-VLS-решетка, mm } & \multicolumn{2}{|c|}{150} \\
\hline \multicolumn{4}{|c|}{ Плоская VLS-решетка } \\
\hline \multicolumn{2}{|l|}{ Апертура, mm } & \multicolumn{2}{|l|}{$50 \times 20$} \\
\hline \multicolumn{2}{|c|}{ Угол отклонения, $^{\circ}$} & \multicolumn{2}{|l|}{32} \\
\hline \multicolumn{2}{|c|}{ Порядок дифракции } & \multicolumn{2}{|c|}{ Первый внешний } \\
\hline \multicolumn{2}{|c|}{ Расстояние решетка-выходная щель, mm } & \multicolumn{2}{|l|}{1200} \\
\hline \multicolumn{2}{|c|}{ Спектральный диапазон, $\AA$} & $125-1000$ & $900-4200$ \\
\hline \multirow{3}{*}{$\begin{array}{l}\text { Коэффициенты } \\
\text { VLS-решетки }\end{array}$} & $p_{0}, \mathrm{~mm}^{-1}$ & 600 & 150 \\
\hline & $p_{1}, \mathrm{~mm}^{-2}$ & 0.956 & 0.239 \\
\hline & $p_{2}, \mathrm{~mm}^{-3}$ & $-1.6 \cdot 10^{-3}$ & $-1.4 \cdot 10^{-3}$ \\
\hline \multicolumn{2}{|c|}{ Обратная линейная дисперсия, $\AA / \mathrm{mm}$} & $3.6-2.3$ & $14-9.1$ \\
\hline \multicolumn{2}{|c|}{ Разрешающая способность } & $>1000$ & $>2000$ \\
\hline
\end{tabular}

$p_{2}=-1.40 \cdot 10^{-4} \mathrm{~mm}^{-3}$, получим значения $R>2000$ во всем спектральном диапазоне 900-4200 А (рис. 4).

Общие параметры рассчитанной схемы VLSмонохроматора приведены в таблице.

\section{Заключение}

Рассчитан проект монохроматора высокого разрешения для спектрального диапазона 125-4200 А, который можно использовать на измерительном канале нового сибирского синхротрона четвертого поколения „СКИФ“ („Сибирский кольцевой источник фотонов“"). Монохроматор по схеме Хеттрика-Андервуда использует вогнутое фокусирующее зеркало и две сменных VLSрешетки. Сканирование по длинам волн осуществляется поворотом решетки при постоянном направлении выхода излучения. Аберрации зеркала скользящего падения, установленного в сильно несимметричной схеме, подавляются VLS-решеткой. Монохроматор способен обеспечить разрешающую способность свыше 1000 в диапазоне 125-1000 А и свыше 2000 в диапазоне 900-4200 АА.

\section{Финансирование работы}

Работа выполнена при частичной поддержке гранта Министерства науки и высшего образования Российской Федерации № 075-15-2020-781.

\section{Конфликт интересов}

Авторы заявляют, что у них нет конфликта интересов.

\section{Список литературы}

[1] A.V. Bukhtiyarov, V.I. Bukhtiyarov, A.D. Nikolenko, I.P. Prosvirin, R.I. Kvon, O.E. Tereshchenko. AIP Conf. Proc., 2299, 060003 (2020). DOI: 10.1063/5.0030740

[2] W.R. Hunter, R.T. Williams, J.C. Rife, J.P. Kirkland, M.N. Kabler. Nucl. Instrum. Meth., 195, 141 (1982). DOI: $10.1016 / 0029-554 \mathrm{X}(82) 90768-6$.

[3] M.C. Hettrick, J.H. Underwood. AIP Conf. Proc., 147, 237 (1986). DOI: 10.1063/1.35993.

[4] M.C. Hettrick, J.H. Underwood, P. Batson, M. Eckart. Appl. Opt., 27 (2), 200 (1988). DOI: 10.1364/AO.27.000200

[5] P. Miotti, N. Fabris, F. Frassetto, C. Spezzani, L. Poletto. AIP Conf. Proc., 2054, 060023 (2019); DOI: 10.1063/1.5084654.

[6] Е.А. Вишняков, А.О. Колесников, А.С. Пирожков, Е.Н. Рагозин, А.Н. Шатохин. Квант. электрон., 48 (10), 916 (2018) [E.A. Vishnyakov, A.O. Kolesnikov, A.S. Pirozhkov, E.N. Ragozin, A.N. Shatokhin. Quant. Electron., 48 (10), 916 (2018)]. DOI: 10.1070/QEL16707

[7] Е.Н. Рагозин, Е.А. Вишняков, А.О. Колесников, А.С. Пирожков, А.Н. Шатохин. УФН, 191 (5), 522 (2021). [E.N. Ragozin, E.A. Vishnyakov, A.O. Kolesnikov, A.S. Pirozhkov, A.N. Shatokhin. Phys. Usp., 191 (5), 522 (2021). DOI: 10.3367/UFNe.2020.06.038799] 
[8] J. Dunn, E.W. Magee, R. Shepherd, H. Chen, S.B. Hansen, S.J. Moon, G.V. Brown, M.-F. Gu, P. Beiersdorfer, M.A. Purvis. Rev. Sci. Instrum., 79, 10E314 (2008).

DOI: $10.1063 / 1.2968704$

[9] T. Harada, H. Sakuma, K. Takahashi, T. Watanabe, H. Hara, T. Kita. Appl. Opt., 37 (28), 6803 (1998). DOI: $10.1364 / A O .37 .006803$

[10] M.C. Hettrick, S. Bowyer. Appl. Opt., 22 (24), 3921 (1983). DOI: $10.1364 / A O .22 .003921$

[11] M.C. Hettrick, S. Bowyer, R.F. Malina, C. Martin, S. Mrowka. Appl. Opt. 24 (12), 1737 (1985). DOI: 10.1364/AO.24.001737

[12] A. Miyake, T. Miyachi, M. Amemiya, T. Hasegawa, N. Ogushi, T. Yamamoto, F. Masaki, Y. Watanabe. Proc. SPIE, 5037, 647 (2003). DOI: $10.1117 / 12.484969$

[13] M. Terauchi, S. Koshiya, F. Satoh, H. Takahashi, N. Handa, T. Murano, M. Koike, T. Imazono, M. Koeda, T. Nagano, H. Sasai, Y. Oue, Z. Yonezawa, S. Kuramoto. Microsc. Microanal., 20, 692 (2014).

DOI: $10.1017 /$ S1431927614000439

[14] J.H. Underwood, E.M. Gullikson, M. Koike, S. Mrowka. Proc. SPIE, 3150, 40 (1997). DOI: 10.1117/12.292734

[15] J.J. Wang, Y.E. Mao, T. Shi, R. Chang, S. Qiao. Chin. Phys. C, 39 (4), 048001 (2015).

[16] L. Du, X. Du, Q. Wang, J. Zhong. Nucl. Instrum. Meth. A, 877, 65 (2018). DOI: 10.1016/j.nima.2017.09.045

[17] O. Fuchs, L. Weinhardt, M. Blum, M. Weigand, E. Umbach, M. Bär, C. Heske, J. Denlinger, Y.-D. Chuang, W. McKinney, Z. Hussain, E. Gullikson, M. Jones, P. Batson, B. Nelles, R. Follath. Rev. Sci. Instrum., 80, 063103 (2009) DOI: $10.1063 / 1.3133704$

[18] T. Warwick, Y.-D. Chuang, D.L. Voronov, H.A. Padmore. J. Synchrotron Radiat., 21, 736 (2014). DOI: $10.1107 / \mathrm{S} 1600577514009692$

[19] J. Dvorak, I. Jarrige, V. Bisogni, S. Coburn, W. Leonhardt. Rev. Sci. Instrum., 87, 115109 (2016). DOI: $10.1063 / 1.4964847$

[20] M.A. Cornu. Comptes Rendus Acad. Sci., 117, 1032 (1893).

[21] T. Harada, S. Moriyama, T. Kita. Jpn. J. Appl. Phys., 14 (51), 175 (1975). DOI:10.7567/JJAPS.14S1.175

[22] T. Harada, T. Kita. Appl. Opt., 19 (23), 3987 (1980). DOI: $10.1364 / \mathrm{AO} .19 .003987$

[23] T. Kita, T. Harada. Appl. Opt., 31 (10), 1399 (1992). DOI: 10.1364/AO.31.001399

[24] T. Kita, T. Harada, N. Nakano, H. Kuroda. Appl. Opt., 22 (4), 512 (1983). DOI: 10.1364/AO.22.000512

[25] Hettrick Scientific [Электронный ресурс] Режим доступа: http://hettrickscientific.com/

[26] А.О. Колесников, Е.А. Вишняков, А.Н. Шатохин, Е.Н. Рагозин. Квант. электрони., 49 (11), 1054 (2019).

[A.O. Kolesnikov, E.A. Vishnyakov, A.N. Shatokhin, E.N. Ragozin. Quant. Electron., 49 (11), 1054 (2019). DOI: 10.1070/QEL17074]

[27] A.N. Shatokhin, A.O. Kolesnikov, P.V. Sasorov, E.A. Vishnyakov, E.N. Ragozin. Opt. Express, 26 (15), 19009 (2018). DOI: 10.1364/OE.26.019009

[28] ESRF. XOP (X-ray Oriented Programs) [Электронный ресурс] Режим доступа: https://www.esrf.fr/Instrumentation/ software/ data-analysis/xop2.3 